\title{
Two-Stage Total Knee Arthroplasty for Prosthetic Joint Infection
}

\author{
Min Seok Cha, $\mathrm{MD}^{1}$, Se Hyun Cho, $\mathrm{MD}^{2}$, Dong Hee Kim, $\mathrm{MD}^{3}$, Hong Kwon Yoon, $\mathrm{MD}^{3}$, Ho Seung Cho, $\mathrm{MD}^{3}$, \\ Dong Yeong Lee, $\mathrm{MD}^{3}$, Sang Hyuk Lee, $\mathrm{MD}^{3}$, and Sun Chul Hwang, $\mathrm{MD}^{3}$ \\ ${ }^{1}$ Department of Orthopaedic Surgery, Saeum Hospital, Seoul; ${ }^{2}$ Department of Orthopaedic Surgery, SKY Hospital, Seoul; ${ }^{3}$ Department of Orthopaedic Surgery, Institute \\ of Health Sciences, Gyeongsang National University Hospital, Jinju, Korea
}

\begin{abstract}
Purpose: This retrospective review was conducted to identify prognostic factors for two-stage reimplantation for infected total knee arthroplasty (TKA) and the rate of reinfection following revision TKA.

Materials and Methods: Out of 88 patients diagnosed with post-TKA infection between 1998 and 2011, 76 underwent two-stage reimplantation and were reviewed in this study. The 76 patients were divided into two groups-those who experienced reinfection and those who did not. Comorbidities, culture results, and inflammation indices were analyzed and compared between the two groups.

Results: Of the 76 patients who underwent a two-stage reimplantation, 18 (23.7\%) experienced reinfection. Patients with more than three comorbidities had significantly higher reinfection rates than those with less than three comorbidities ( $47.1 \%$ vs. $4.8 \%$, $\mathrm{p}=0.032)$. The reinfection rate between the culture positive prosthetic joint infection group and the culture negative prosthetic joint infection group was not significantly different $(\mathrm{p}=0.056)$. Inflammation indices (erythrocyte sedimentation rate [ESR] and C-reactive protein [CRP]) showed a statistically significant difference between patients with reinfection and those without reinfection at 4 weeks after the first-stage surgery.

Conclusions: Reimplantation must be carefully performed when the risk of reinfection is high, particularly in patients with more than three systemic or local comorbidities and higher inflammation indices (ESR and CRP) prior to revision TKA.
\end{abstract}

Keywords: Knee, Arthroplasty, Infection, Two-stage revision, Comorbidities

\section{Introduction}

Total knee arthroplasty (TKA) relieves knee pain and restores joint function and quality of life.

However, infection after TKA is a severe complication that often requires surgical intervention and antibiotic treatment ${ }^{1-4)}$. Two-stage reimplantation is a widely used surgical intervention for infected TKA cases; in these cases antibiotics are selected

Received May 26, 2014; Revised (1st) August 24, 2014;

(2nd) December 23, 2014; (3rd) April 13, 2015; Accepted May 4, 2015

Correspondence to: Sun Chul Hwang, MD

Department of Orthopaedic Surgery, Gyeongsang National University

School of Medicine, 79 Gangnam-ro, Jinju 660-702, Korea

Tel: +82-55-750-8102, Fax: +82-55-761-9477

E-mail: hscspine@hanmail.net

This is an Open Access article distributed under the terms of the Creative Commons Attribution Non-Commercial License (http://creativecommons.org/licenses/by-nc/4.0/) which permits unrestricted non-commercial use, distribution, and reproduction in any medium, provided the original work is properly cited. and administered for 2-6 weeks, depending on the cultured microorganisms ${ }^{1,5,6)}$. Despite aggressive treatment, success rates for this method range from $37.1 \%$ to $100 \%$ depending on the patient's immunological status, the cultured microorganism, and general or local comorbidities of the patient ${ }^{6-10)}$. The use of twostage reimplantation is controversial due to discrepancies in the reported influence of systemic and local comorbidities on postTKA infection. For example, some reports show that general comorbidities affect reinfection ${ }^{11,12)}$, whereas others suggest that systemic comorbidities do not directly affect the reinfection rate ${ }^{13,14)}$. Although there is abundant research on how the reinfection ratio is affected by specific microorganisms ${ }^{12,13,15,16)}$, few studies address reinfection ratios in culture negative prosthetic joint infections ${ }^{17,18)}$. Additionally, the usefulness of inflammation indices (erythrocyte sedimentation rate [ESR] and C-reactive protein [CRP]) in predicting the rate of reinfection remains controversial $^{19,20)}$. Prosthetic joint infections have increased in prevalence over the past few decades; however, despite the importance of this complication, only a few studies have investigated post-TKA 
infection $^{21-23)}$.

In this study, we identified the prognostic factors for two-stage reimplantation performed to treat post-TKA infection and the rate of reinfection. We hypothesized 1) that reinfection rates would increase in patients with systemic or local comorbidities, 2 ) that rates of reinfection would be higher in culture negative cases due to the difficulty of using effective antibiotics, 3) and that higher hematological inflammation indices (ESR and CRP) would affect the reinfection rates.

\section{Materials and Methods}

\section{Study Group}

Our investigation was performed after receiving approval from the Institutional Review Board of our hospital. Out of 88 patients, we selected 76 subjects diagnosed with infection and treated by the two-stage reimplantation following TKA from January 1998 to January 2011. Of the total 88 patients, 6 did not satisfy diagnostic criteria, 4 did not perform the last follow-up visit, 1 underwent a limb amputation due to necrotizing fasciitis, and 1 died of exacerbation of an underlying disease; these patients were excluded. All operations were performed by a single surgeon (Senior Hwang). The average age of the patients was 66.50 years (range, 53 to 84 years) and there were 19 males and 57 females. The average observation period was 30.3 months (range, 24.2 to
80.6 months). The following parameters were recorded for each subject: height, weight, body mass index (BMI), general and local comorbidities, the inflammation indices (ESR and CRP), culture test results, and the medical record of the patient after surgical treatment prior to reimplantation and then for the duration of antibiotic treatment. These data were compared and analyzed between the patients with reinfection and those without reinfection.

\section{Definition of Periprosthetic Infection, Culture Negative Periprosthetic Infection, Treatment Failure after TKA, and Radiological Evaluation}

The patient groups were classified based on the diagnostic standard of infection after the artificial joint implantation proposed by the Musculoskeletal Infection Society (MSIS) ${ }^{24)}$, based on the definition of treatment failure proposed by Marculescu et $\mathrm{al}^{25}{ }^{25}$, and based on the diagnosis standard for the culture negative prosthetic joint infection proposed by Osmon et al. ${ }^{1)}$ (Table 1). Patients with more than three systemic or local comorbidities were assigned to the high-risk group and patients with less than three systemic or local comorbidities were assigned to the low-risk group, as described by the Cierny classification system ${ }^{14)}$. All the radiological records of the patients, including anteroposterior and lateral images of the knee, were retrospectively reviewed based on the Knee Society's TKA roentgenographic evaluation and scoring system proposed by Ewald ${ }^{26)}$. The data were tabulated for

Table 1. Definition of Terms for the Study of Prosthetic Joint Infection (PJI)

A new definition of PJI

Definition of PJI

1. A sinus tract communicates with the prosthesis

2. A pathogen is isolated by culture from two separate tissue or fluid samples obtained from the affected prosthetic joint

3. Four of the following six criteria exist

a. Elevated serum erythrocyte sedimentation rate or serum C-reactive protein concentration

b. Elevated synovial white blood cell count

c. Elevated synovial neutrophil percentage

d. Presence of purulence in the affected joint

e. Isolation of a microorganism in one culture of periprosthetic tissue or fluid

f. Greater than five neutrophils per high-power field in five high-power field, observed by histologic analysis of periprosthetic tissue at 400 times magnification

Definition of culture negative PJI

Joint aspiration or intraoperative specimens are negative for aerobic and anaerobic bacteria cultures and purulence surrounds the prosthesis; acute inflammation is visible in histopathologic examination at the time of surgery; or sinus tract communication with the prosthesis, with or without prior use of antimicrobials, occurs.

Definition of treatment failure

1. Occurrence of PJI resulting from the original microorganism at any time after the surgical procedure (relapse)

2. Occurrence of PJI resulting from a different strain or microorganism (reinfection) at any time after the surgical procedure

3. Presence of acute inflammation in the periprosthetic tissue by histopathologic examination or after a subsequent surgery in the joint

4. Development of a sinus tract

5. Death from prosthesis-related infection or indeterminate clinical failure 


\section{Cha et al. Two-Stage Revision TKA for Prosthetic Joint Infection}

each component based on the width and the extent of associated radiolucent depth in millimeters for each zone.

\section{Sequential Reimplantation and Antibiotic Treatment}

All patients diagnosed with infection had artificial joints and bone cement removed. The cement was meticulously removed by three-phase debridement of the bone surfaces, beginning with rongeurs, followed by curettes, and completed with a high-torque reamer to burr away all surfaces exposed to the cement. Next, the infected tissues were delicately debrided and an antibiotic combined with the cement spacer ( $5 \mathrm{~g}$ of gentamicin, $1 \mathrm{~g}$ of vancomycin, and $1 \mathrm{~g}$ of ceftriaxone per $40 \mathrm{~g}$ of cement) was inserted. A full cementing technique was employed to cement the tibial and femoral components (cementation of both the undersurface and the stem). The combined antibiotic regimens were selected by taking into consideration of the data from our institute on high frequency multidrug-resistant hospital acquired microorganisms. Antibiotics specific to the cultured microorganisms were intravenously injected and oral antibiotics were administered, if required, after the surgery. Culture negative prosthetic joint infection patients were injected with vancomycin. Two-stage reimplantation was performed after the hematological and radiological signs of infection remission were observed. All the patients with revision implantation underwent the debridement of necrotic tissue once more. We treated all knees using the same protocol and the same revision instrument with nonporous, fluted, diaphyseal-engaging titanium stems (NexGen Legacy Constrained Condylar Knee; Zimmer Inc., Warsaw, IN, USA). An antibiotic combined with cement ( $1 \mathrm{~g}$ of vancomycin per $40 \mathrm{~g}$ of cement) was used to fix the implants into the bone and fill the bone defects.

\section{Statistical Analyses}

Independent t-tests were used to compare the demographic and treatment groups by analyzing the inflammation indices (ESR and CRP) after the insertion of the antibiotic-combined cement spacer at 1,2, and 4 weeks, and prior to the reimplantation. Reinfection rates were compared and analyzed by positive and negative culture depending on the risk group. Chi-square cross analyses were performed to compare the reinfection rates of each group. Logistic regression analysis was performed to investigate the factors responsible for reinfection in the high-risk group, identification of microorganisms, the time period for antibiotic

Table 2. Demographics of Reinfection Group and Non-Reinfection Group

\begin{tabular}{|c|c|c|c|}
\hline Parameter & $\begin{array}{l}\text { Reinfection group } \\
\qquad(\mathrm{n}=18)\end{array}$ & $\begin{array}{l}\text { Non-reinfection group } \\
\qquad(\mathrm{n}=58)\end{array}$ & p-value \\
\hline Age (yr) & $68.1 \pm 7.1(55-81)$ & $66.0 \pm 7.1(53-84)$ & 0.272 \\
\hline $\operatorname{Sex}(M: F)$ & $3: 15$ & $16: 42$ & 0.350 \\
\hline Height (m) & $1.6 \pm 0.1(1.5-1.7)$ & $1.6 \pm 0.1(1.5-1.7)$ & 0.196 \\
\hline Weight (kg) & $58.8 \pm 7.9(50-75)$ & $59.2 \pm 7.2(47-77)$ & 0.850 \\
\hline BMI $\left(\mathrm{kg} / \mathrm{m}^{2}\right)$ & $22.5 \pm 1.8(20.0-25.4)$ & $22.1 \pm 1.7(18.8-26.6)$ & 0.384 \\
\hline Revision interval (wk) & $15.6 \pm 4.9(4-174)$ & $14.7 \pm 4.1(5-126)$ & 0.707 \\
\hline Period of antibiotic treatment (day) & $56.1 \pm 20.5(49-199)$ & $60.6 \pm 18.6(26-172)$ & 0.183 \\
\hline Follow-up period (mo) & $29.2 \pm 15.3(24-56)$ & $33.4 \pm 8.1(24-80)$ & 0.000 \\
\hline
\end{tabular}

Values are presented as mean \pm standard deviation or number (range).

BMI: body mass index.

Table 3. Presence of Radiolucency in the Knees with Periprosthetic Infection according to Radiographic View and Zone of Implant

\begin{tabular}{|c|c|c|c|c|c|c|c|}
\hline \multirow{2}{*}{ Radiograph } & \multicolumn{7}{|c|}{ Zone } \\
\hline & 1 & 2 & 3 & 4 & 5 & 6 & 7 \\
\hline \multicolumn{8}{|l|}{ Tibia } \\
\hline Anteroposterior view & $8(29.6)$ & $3(11.1)$ & $2(7.4)$ & $4(14.8)$ & $0(0)$ & $0(0)$ & $0(0)$ \\
\hline Lateral view & $4(14.8)$ & $2(7.4)$ & $0(0)$ & & & & \\
\hline \multicolumn{8}{|l|}{ Femur } \\
\hline Lateral view & $2(7.4)$ & $0(0)$ & $0(0)$ & $2(7.4)$ & $0(0)$ & $0(0)$ & $0(0)$ \\
\hline
\end{tabular}

Values are presented as number (\%). 
use, age, CRP levels, and ESR. SPSS ver. 18.0 (SPSS Inc., Chicago, IL, USA) was used for statistical analyses and statistical significance was defined as a p-value of less than 0.05 .

\section{Results}

\section{Demographic and Radiographic Data}

Of the 76 patients who underwent a two-stage reimplantation, 58 individuals $(76.3 \%)$ had no reinfection and 18 individuals (23.7\%) had reinfection after the two-stage reimplantation. No significant differences were found between the two groups with regard to age, gender, height, weight or BMI, or treatment method, the different time period between surgical debridement and reimplantation, and the extent of antibiotic use (Table 2). High rates of radiolucent areas were observed in the knees of the patients at the time of the diagnosis of periprosthetic infection (Table 3).

Table 4. Logistic Regression Analysis of the Influence of Comorbidities on Reinfection

\begin{tabular}{lcc}
\hline \multicolumn{1}{c}{ Variable } & $\begin{array}{c}\text { Odds ratio } \\
(95 \% \mathrm{CI})\end{array}$ & p-value \\
\hline Comorbidities (more than three) & $3.27(2.701-3.839)$ & 0.037 \\
ESR $(\mathrm{mm} / \mathrm{hr})$ & $1.34(1.212-1.468)$ & 0.037 \\
CRP $(\mathrm{mg} / \mathrm{L})$ & $1.04(1.022-1.058)$ & 0.031 \\
\hline
\end{tabular}

CI: confidence interval, ESR: erythrocyte sedimentation rate, CRP: C-reactive protein.

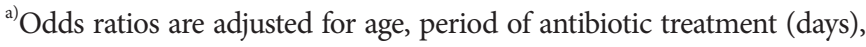
culture results, and duration of follow-up.
2. Comparison of Reinfection Rates according to Comorbidities, Culture Test Results, and Inflammation Indices

The risk of reinfection was higher in those with the more sys-

Table 5. Systemic and Local Comorbidities according to the Cierny Classification

\begin{tabular}{lccc}
\hline \multicolumn{1}{c}{ Comorbidities } & $\begin{array}{c}\text { Reinfection } \\
(\mathrm{n}=18)\end{array}$ & $\begin{array}{c}\text { Non-reinfection } \\
(\mathrm{n}=58)\end{array}$ & p-value \\
\hline Systemic comorbidities & 8 & 18 & 0.233 \\
Age $>70$ (yr) & 8 & 16 & 0.384 \\
Obesity (BMI>23 kg/m $\left.{ }^{2}\right)$ & 12 & 10 & 0.000 \\
Diabetes & 0 & 2 & 0.425 \\
Steroid therapy & 0 & 2 & 0.325 \\
Tumor & 0 & 2 & 0.425 \\
Alcohol abuse & 4 & 3 & 0.210 \\
Chronic hypoxia & 4 & 0 & 0.000 \\
Rheumatoid arthritis & 8 & 14 & 0.077 \\
HTN ${ }^{\text {a }}$ & 4 & 2 & 0.010 \\
CKD & & & \\
Local comorbidities & & 0 & - \\
Chronic lymphedema & 0 & 3 & 0.949 \\
Venous stasis & 1 & 3 & 0.375 \\
Phlebitis & 2 & 0 & - \\
Peripheral arterial disease & 0 & 2 & 0.375 \\
Extensive scarring & 3 & 0 & - \\
Post-radiation fibrosis & 0 & & \\
\hline
\end{tabular}

BMI: body mass index, HTN: hypertension, CKD: chronic kidney disease. ${ }^{a}$ HTN and CKD are originally excluded from Cierny classification but included in our study as comorbidities.

Table 6. Identified Microorganisms and Administered Antibiotics

\begin{tabular}{llcc}
\multicolumn{1}{c}{ Organism } & \multicolumn{1}{c}{ Antibiotics } & N & Percent \\
\hline Staphylococcus aureus & Cephalosporin or nafcillin or vancomycin \pm ripamfin & 17 & 22.4 \\
Staphylococcus epidermidis & Cephalosporin or nafcillin or vancomycin \pm ripamfin & 11 & 14.5 \\
Staphylococcus lugdunensis & Cephalosporin or nafcillin or vancomycin \pm ripamfin & 1 & 1.3 \\
Steptococcus species & Cephalosporin or penicillin or ampicillin+sulbactam or vancomycin & 3 & 3.9 \\
Enterococcus species & Cephalosporin or penicillin or vancomycin & 4 & 3.3 \\
Pseudomonas aerusinosa & Piperacillin \& tobramycin & 3 & 3.9 \\
Escherachia coli & Cephalosporin & 1 & 1.3 \\
Serratia marcescens & Cephalosporin & 1 & 1.3 \\
MRSA & Vancomycin \pm ripamfin or teicoplanin or linezolid \pm ripamfin & 2 & 13.2 \\
MRSE & Vancomycin \pm ripamfin or teicoplanin or linezolid \pm ripamfin & 2 & 2.6 \\
Multiple organisms & Vancomycin & 29 & 1.3 \\
Culture negative & Vancomycin \pm rifampin & 29.0 \\
\hline
\end{tabular}

MRSA: methicillin-resistant Staphylococcus aureus, MRSE: methicillin-resistant Staphylococcus epidermidis. 
A

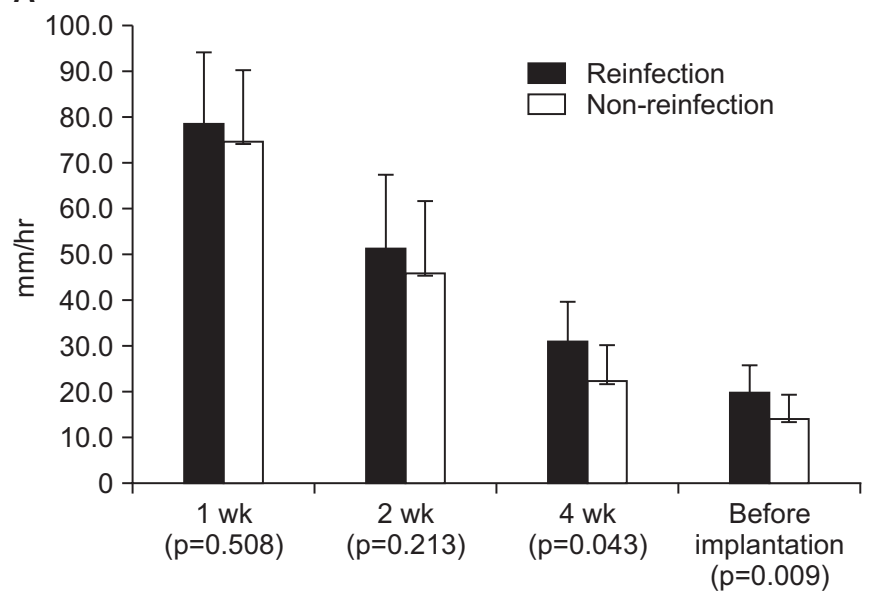

B

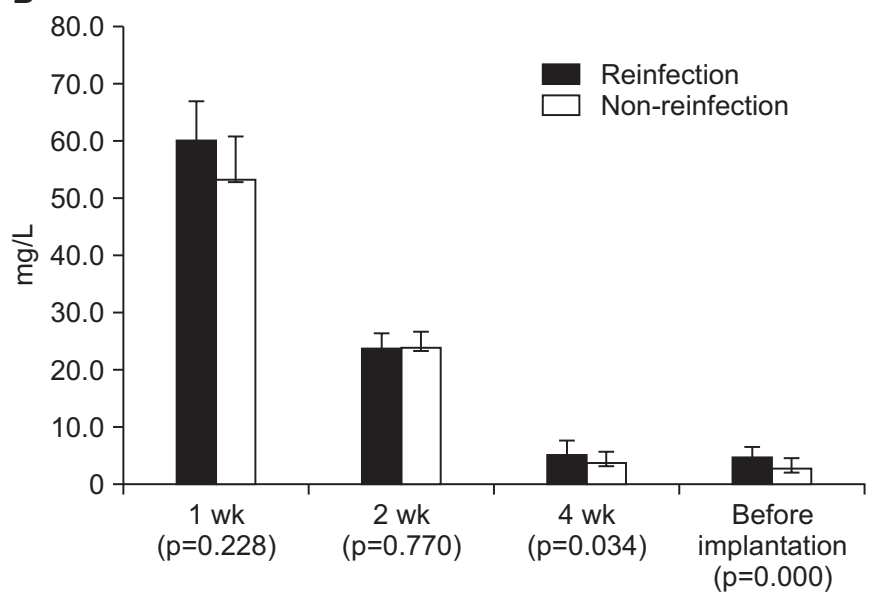

Fig. 1. The erythrocyte sedimentation rate (A) and C-reactive protein level (B) were compared between the reinfection group and the non-reinfection group.

temic and local comorbidities and higher inflammation indices (ESR and CRP) determined by the logistic regression analysis (Table 4). Of the 76 patients that underwent the reimplantation, 36 had systemic comorbidities, 22 had hypertension, 22 had diabetes, 2 had liver disease, 6 had renal disease, 2 had Cushing syndrome, 2 had tumors, 7 had chronic obstructive pulmonary disease, and 4 had rheumatoid arthritis. The local comorbidities in the patients consisted of deep vein thrombosis (4 patients), phlebitis (5 patients), and scars around the surgical area (5 patients) (Table 5). Of the 34 high-risk patients with more than three systemic or local comorbidities, 16 experienced reinfection (47.1\%). Out of the 42 patients in the low-risk group, 2 experienced reinfection (4.8\%). The high-risk group had a significantly higher reinfection rate than the low-risk group ( $\mathrm{p}=0.032)$. Staphylococcus aureus (S. aureus) was cultured in 17 patients (22.4\%) and Staphylococcus epidermidis was cultured in 11 patients (14.5\%). Methicillin-resistant Staphylococcus aureus (MRSA) was cultured in 10 patients (13.2\%) and 22 patients (29\%) had negative culture tests (Table 6). Microorganisms were identified in 16 out of 18 patients $(88.9 \%)$ with reinfection after the reimplantation, and in 38 out of 58 patients $(65.5 \%)$ without reinfection after reimplantation. There was no statistical difference in reinfection rates depending on the culture test results ( $\mathrm{p}=0.056)$. Inflammation indices (ESR and CRP), measured at 1,2, and 4 weeks after the first-stage operation and before the reimplantation, were significantly higher in the reinfection group than in the group without reinfection at 4 weeks and before reimplantation: 30.7 vs. $22.1(\mathrm{p}=0.043)$ and 5.4 vs. 4.1 ( $\mathrm{p}=0.034)$, respectively, after the first-stage operation and 19.3 vs. $13.7,(\mathrm{p}=0.009)$ and 5.0 vs. $3.1(\mathrm{p}<0.05)$, respectively, before reimplantation (Fig. 1).

\section{Discussion}

One of the most important findings of this study is that the high-risk group with more than three systemic and local comorbidities had high reinfection rates after the two-stage reimplantation. Another important finding is that the reinfection group showed significantly higher ESR and CRP at 4 weeks after the first-stage operation and before reimplantation than the non-infection group. However, the risk of reinfection was not significantly different between groups with different culture results after the two-stage reimplantation. In accordance with our hypothesis predicting that reinfection rates would increase with the presence of more systemic or local comorbidities in patients undergoing two-stage reimplantation, we showed that the highrisk group had higher reinfection rates $(\mathrm{p}=0.032)$. The prevalence of diabetes ( $67 \%$ vs. $17 \%, \mathrm{p}<0.001)$, rheumatoid arthritis ( $22 \%$ vs. $0 \%, \mathrm{p}<0.001)$, and chronic kidney diseases ( $22 \%$ vs. $3 \%, \mathrm{p}=0.010)$ showed statistically significant differences between the reinfection and non-reinfection groups. These comorbidities also have clinical significance, which requires careful attention prior to and following the surgery. Jamsen et al. ${ }^{11)}$ stated that high blood glucose level before reimplantation is an important risk factor in patients who are not diagnosed as obese or diabetic. Recently, however, Tigani et al. ${ }^{14)}$ mentioned that a single systemic comorbidity does not affect the reinfection rates, but three or more systemic and local comorbidities result in higher reinfection rates. Kubista et al. ${ }^{13)}$ also reported that diabetic patients experienced higher reinfection rates, but the difference was not statistically significant. It remains controversial whether systemic or local comorbidities affect reinfection; however, our study showed that the high-risk 
group with more than three systemic or local comorbidities experienced higher reinfection rates than the low-risk group. Therefore, it is not only essential to manage systemic comorbidities, but also to pay deliberate attention to the local comorbidities.

Our second hypothesis predicted that without microorganism identification, patients would show higher reinfection rates due to the possibility of using nonspecific antibiotics; however, our results indicate that culture test results do not affect the reinfection rates. Previous studies reported that it is difficult to treat infected reimplantations due to drug resistant microorganisms ${ }^{15,16)}$. However, Kubista et al. ${ }^{13)}$ reported that there was no difference in reinfection rates between those infected with drug resistant bacteria and those infected with non-resistant bacteria. They also showed that treatment with cefazolin resulted in lower reinfection rates thanthe treatment with vancomycin, because vancomycin is less potent than cefazolin. Cierny and DiPasquale ${ }^{12)}$ reported that the virulence of microorganisms does not affect reinfection rates. Several studies address the effect of microorganisms on reinfection rates; however, the risk for reinfection depending on the identification of microorganism is less commonly reported. In fact, there is no standard treatment protocol for culture negative prosthetic joint infections; therefore, cephalosporin, which targets S. aureus, the most common pathogenic microorganism, is used; otherwise, vancomycin, a glycopeptide, is used when nosocomial multi-drug resistant microorganisms are present after long-term antibiotic therapy ${ }^{27}$. Alternatively, an organism-specific antibiotic is used for positive culture results. Culture negative prosthetic joint infections are diagnosed by standards proposed by Berbari et al. ${ }^{17)}$ of the Infectious Disease Society of America; however, there are no standardized protocols for antibiotic treatment of culture negative prosthetic joint infections. In fact, the Berbari et al. ${ }^{17)}$ study focused only on the efficacy of using vancomycin in drug resistant and combined bacterial infections. Another study by Marschall et al. ${ }^{28)}$ used intravenous vancomycin injection in $79 \%$ of the patients with culture negative prosthetic joint infections. They also used vancomycin for single intravenous antibiotic treatment in order to target commonly identified S. aureus and MRSA, and in the case of combined intravenous antibiotic treatment, they combined vancomycin with quinolones, which are effective for treating gram negative bacteria. Marschall et al. ${ }^{28)}$ defined the period of antibiotic usage based on the blood test results and clinical symptoms, and found that the period of usage between the culture positive group (mean, $60.8 \pm 23.3$ days) and the culture negative group (mean, $58.8 \pm 27.8$ days) was not statistically different. In this study, comparison of the rate of reinfection depending on culture test results indicated no difference in reinfection between the group using vancomycin and the group given sensitive antibiotics $(\mathrm{p}=0.056)$.

Even though many methods have been used to diagnose infection after prosthetic joint implantation, a clear diagnosis, which may assist in decision-making prior to the operation, is difficult to obtain. Recent studies report that inflammation indices (ESR and CRP) increase after TKA and become normalized between roughly 3 weeks to 2 months ${ }^{29)}$. Levitsky et al. ${ }^{20)}$ suggested that CRP should be used to predict infection because it has higher sensitivity and specificity than ESR, the more widely used diagnostic measure. In this study, we measured ESR and CRP using a hematologic test at the time of the primary operation before reimplantation and at 1,2, and 4 weeks before the reimplantation. The inflammatory indices of 76 patients gradually decreased from the time of the primary operation until the second reimplantation. In addition, each set of indices measured 4 weeks after and before the first-stage operation presented statistically significant differences between the group with successful treatment and the group with the reinfection. This indicates that the inflammation indices (ESR and CRP) at 4 weeks after the first-stage operation and before reimplantation were significantly higher in the group with failed treatment, and that the incidence of reinfection increased. This confirms our final hypothesis that the reinfection rates are higher in individuals with high inflammatory indices measured in the antibiotic-combined cement spacer filling for reimplantation. Unfortunately, it is difficult to judge the infection remission by merely comparing inflammation indices due to the small differences in the absolute figures, which do not possess clinical significance despite statistical significance of inflammation indices between the two groups. Therefore, it is most effective to judge after the comparison and analysis of several measurements by considering the various factors that may affect reinfection rate. Inflammatory indices should be considered as a univariate factor.

There are several limitations to consider in the present study. First, this study is a retrospective analysis which, despite the authors' efforts, has a certain amount of selection bias. Second, it was difficult to perform precise statistical analyses due to the small sample size of the group with reinfection after two-stage reimplantation; this is due to the retrospective study design and because surgeries were performed at a single center. For more precise results, large-scale studies need to be performed by multicenter organizations. Third, this study failed to completely rule out the possibility of reinfection, which may occur later than the short period of observation performed here. Last, we do not propose clear diagnostic standards for infection remission in the infected prosthetic joint, and do not compare the clinical function 
of the prosthetic joint after the two-stage reimplantation.

\section{Conclusions}

Reimplantation must be carefully performed when the risk of reinfection is high, particularly in patients with more than three systemic or local comorbidities and higher inflammation indices (ESR and CRP) prior to revision TKA. Our study can aid surgeons in counseling patients regarding their prognosis when faced with the two-stage reimplantation for infected TKA, and provide a basis for future studies.

\section{Conflict of Interest}

No potential conflict of interest relevant to this article was reported.

\section{References}

1. Osmon DR, Berbari EF, Berendt AR, Lew D, Zimmerli W, Steckelberg JM, Rao N, Hanssen A, Wilson WR; Infectious Diseases Society of America. Diagnosis and management of prosthetic joint infection: clinical practice guidelines by the Infectious Diseases Society of America. Clin Infect Dis. 2013;56:e1-25.

2. Zimmerli W, Trampuz A, Ochsner PE. Prosthetic-joint infections. N Engl J Med. 2004;351:1645-54.

3. Darouiche RO. Treatment of infections associated with surgical implants. N Engl J Med. 2004;350:1422-9.

4. Sia IG, Berbari EF, Karchmer AW. Prosthetic joint infections. Infect Dis Clin North Am. 2005;19:885-914.

5. Kurd MF, Ghanem E, Steinbrecher J, Parvizi J. Two-stage exchange knee arthroplasty: does resistance of the infecting organism influence the outcome? Clin Orthop Relat Res. 2010;468:2060-6.

6. Goldman RT, Scuderi GR, Insall JN. 2-stage reimplantation for infected total knee replacement. Clin Orthop Relat Res. 1996;(331):118-24.

7. Haleem AA, Berry DJ, Hanssen AD. Mid-term to long-term followup of two-stage reimplantation for infected total knee arthroplasty. Clin Orthop Relat Res. 2004;(428):35-9.

8. McLaren AC, Spooner CE. Salvage of infected total knee components. Clin Orthop Relat Res. 1996;(331):146-50.

9. Mittal Y, Fehring TK, Hanssen A, Marculescu C, Odum SM, Osmon D. Two-stage reimplantation for periprosthetic knee infection involving resistant organisms. J Bone Joint Surg
Am. 2007;89:1227-31.

10. Hirakawa K, Stulberg BN, Wilde AH, Bauer TW, Secic M. Results of 2-stage reimplantation for infected total knee arthroplasty. J Arthroplasty. 1998;13:22-8.

11. Jamsen E, Nevalainen P, Eskelinen A, Huotari K, Kalliovalkama J, Moilanen T. Obesity, diabetes, and preoperative hyperglycemia as predictors of periprosthetic joint infection: a single-center analysis of 7181 primary hip and knee replacements for osteoarthritis. J Bone Joint Surg Am. 2012;94:e101.

12. Cierny G 3rd, DiPasquale D. Periprosthetic total joint infections: staging, treatment, and outcomes. Clin Orthop Relat Res. 2002;(403):23-8.

13. Kubista B, Hartzler RU, Wood CM, Osmon DR, Hanssen $\mathrm{AD}$, Lewallen DG. Reinfection after two-stage revision for periprosthetic infection of total knee arthroplasty. Int Orthop. 2012;36:65-71.

14. Tigani D, Trisolino G, Fosco M, Ben Ayad R, Costigliola P. Two-stage reimplantation for periprosthetic knee infection: Influence of host health status and infecting microorganism. Knee. 2013;20:9-18.

15. Mortazavi SM, Molligan J, Austin MS, Purtill JJ, Hozack WJ, Parvizi J. Failure following revision total knee arthroplasty: infection is the major cause. Int Orthop. 2011;35:1157-64.

16. Parvizi J, Azzam K, Ghanem E, Austin MS, Rothman RH. Periprosthetic infection due to resistant staphylococci: serious problems on the horizon. Clin Orthop Relat Res. 2009;467:1732-9.

17. Berbari EF, Marculescu C, Sia I, Lahr BD, Hanssen AD, Steckelberg JM, Gullerud R, Osmon DR. Culture-negative prosthetic joint infection. Clin Infect Dis. 2007;45:1113-9.

18. Choi HR, Kwon YM, Freiberg AA, Nelson SB, Malchau H. Periprosthetic joint infection with negative culture results: clinical characteristics and treatment outcome. J Arthroplasty. 2013;28:899-903.

19. Windsor RE, Insall JN, Urs WK, Miller DV, Brause BD. Twostage reimplantation for the salvage of total knee arthroplasty complicated by infection: further follow-up and refinement of indications. J Bone Joint Surg Am. 1990;72:272-8.

20. Levitsky KA, Hozack WJ, Balderston RA, Rothman RH, Gluckman SJ, Maslack MM, Booth RE Jr. Evaluation of the painful prosthetic joint. Relative value of bone scan, sedimentation rate, and joint aspiration. J Arthroplasty. 1991;6:237-44.

21. Puhto AP, Puhto TM, Niinimaki TT, Leppilahti JI, Syrjala HP. Two-stage revision for prosthetic joint infection: out- 
come and role of reimplantation microbiology in 107 cases. J Arthroplasty. 2014;29:1101-4.

22. Silvestre A, Almeida F, Renovell P, Morante E, Lopez R. Revision of infected total knee arthroplasty: two-stage reimplantation using an antibiotic-impregnated static spacer. Clin Orthop Surg. 2013;5:180-7.

23. Bejon P, Berendt A, Atkins BL, Green N, Parry H, Masters S, McLardy-Smith P, Gundle R, Byren I. Two-stage revision for prosthetic joint infection: predictors of outcome and the role of reimplantation microbiology. J Antimicrob Chemother. 2010;65:569-75.

24. Hozack WJ, Parvizi J. New definition for periprosthetic joint infection. J Arthroplasty. 2011;26:1135.

25. Marculescu CE, Berbari EF, Hanssen AD, Steckelberg JM, Harmsen SW, Mandrekar JN, Osmon DR. Outcome of prosthetic joint infections treated with debridement and reten- tion of components. Clin Infect Dis. 2006;42:471-8.

26. Ewald FC. The Knee Society total knee arthroplasty roentgenographic evaluation and scoring system. Clin Orthop Relat Res. 1989;(248):9-12.

27. Moran E, Masters S, Berendt AR, McLardy-Smith P, Byren I, Atkins BL. Guiding empirical antibiotic therapy in orthopaedics: The microbiology of prosthetic joint infection managed by debridement, irrigation and prosthesis retention. J Infect. 2007;55:1-7.

28. Marschall J, Lane MA, Beekmann SE, Polgreen PM, Babcock HM. Current management of prosthetic joint infections in adults: results of an Emerging Infections Network survey. Int J Antimicrob Agents. 2013;41:272-7.

29. Chun KC, Kim KM, Chun CH. Infection following total knee arthroplasty. Knee Surg Relat Res. 2013;25:93-9. 